Case Report

\title{
Extensive Bone Marrow Necrosis: Initial Presentation in Sickle Cell Anemia-A Case Report and Review of the Literature
}

\author{
Sameera A. Alsafwani, ${ }^{1}$ Abdulwahed Al-Saeed, ${ }^{2}$ and Rehab Bukhamsin ${ }^{3}$ \\ ${ }^{1}$ Qatif Central Hospital (QCH), Qatif, Saudi Arabia \\ ${ }^{2}$ Dammam Medical Complex (DMC), Dammam, Saudi Arabia \\ ${ }^{3}$ Dammam Regional Laboratory and Blood Bank (DRL), Dammam, Saudi Arabia \\ Correspondence should be addressed to Sameera A. Alsafwani; sameeraalsafwani@gmail.com
}

Received 7 February 2017; Revised 25 April 2017; Accepted 22 May 2017; Published 13 June 2017

Academic Editor: Akimichi Ohsaka

Copyright (C) 2017 Sameera A. Alsafwani et al. This is an open access article distributed under the Creative Commons Attribution License, which permits unrestricted use, distribution, and reproduction in any medium, provided the original work is properly cited.

\begin{abstract}
Bone marrow necrosis (BMN) is a rare clinical entity that was first described in an autopsy of a sickle cell disease (SCD) patient and is defined as ill-defined necrotic cells in an amorphous eosinophilic background with preservation of cortical bone. The pathophysiology of BMN is not well known; however, occlusion of the bone marrow microcirculation with subsequent hypoxia and cell injury has been thought to be common underlying features. Malignancy has been identified to be the primary cause in $90 \%$ of the cases whereas SCD was found in only $2 \%$. In this report we present an unusual case of SCD with late onset of the disease whose initial presentation was extensive BMN. The patient was not known previously to have SCD, when suddenly she presented with severe cytopenias and marked elevation in serum lactate dehydrogenase (LDH). Bone marrow examination was done to exclude bone marrow infiltration, and BMN with dilated marrow sinuses full of irreversibly sickled cells were the unexpected findings. Patients with a mild SCD phenotype are at high risk of BMN. Thus, a high index of suspicion must be borne in mind, particularly in an area of high SCD prevalence, to recognize and prevent this catastrophic complication.
\end{abstract}

\section{Introduction}

Bone marrow necrosis (BMN) is rarely encountered in clinical practice. It was first described in an autopsy of a sickle cell disease (SCD) patient by Wade and Stevenson [1]. BMN refers to necrosis of myeloid tissue and medullary stroma in large areas of the haemopoietic bone marrow that results in an amorphous eosinophilic background, illdefined necrotic cells with preservation of the cortical bone [2]. The bone marrow trephine shows disruption of normal marrow architecture with loss of fat spaces but generally with preservation of the specular architecture [3]. The incidence of BMN varies among different reports, ranging from 0.3 to $37 \%$ [3]. Malignancy has been identified to be the primary cause of BMN in more than $90 \%$ of the cases [4]. Other nonmalignant causes include hemoglobinopathies, infections, drugs, anorexia nervosa, hemolytic uremic syndrome (HUS), antiphospholipid syndrome, and disseminated intravascular coagulopathy (DIC) $[3,5,6]$. SCD was found to be the primary cause in only $2 \%$ of the cases [4]. Although the pathophysiology of BMN is not well defined, occlusion of the bone marrow microcirculation is assumed to be the initiating factor [7]. Occlusion of the microcirculation could be caused by variable factors such as tumor cell emboli, fibrin thrombi, toxic effect of drugs, radiation, bacterial infection, or cytokines $[2,8,9]$. Patients with extensive BMN usually present with fever, bone pain, and fatigue and have pancytopenia with a leucoerythroblastic picture in the peripheral blood film (PBF) and, characteristically, a striking number of nucleated red blood cells (NRBCs) [10]. Elevated serum lactate dehydrogenase ( $\mathrm{LDH})$, alanine transferase (ALT), alkaline phosphates (ALP), and uric acid levels are also common features [4]. Examination of bone marrow biopsy is a prerequisite for the accurate diagnosis of BMN. We report an unusual case of a 26-year-old Saudi female whose bone marrow was referred for evaluation due to her clinical presentation with generalized body aches, jaundice, hepatosplenomegaly with anemia, and thrombocytopenia. 


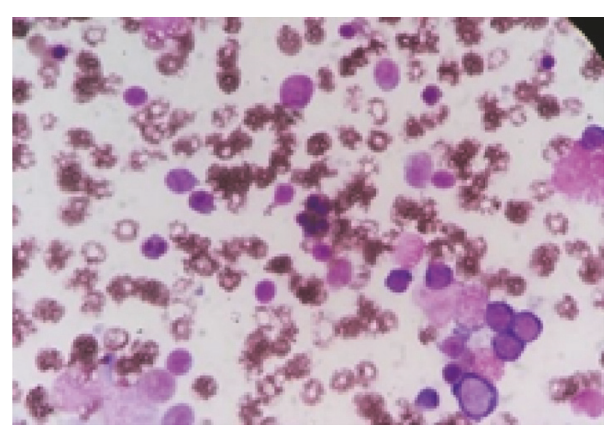

(a)

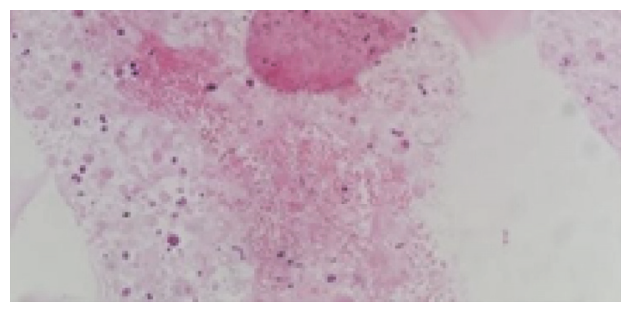

(c)

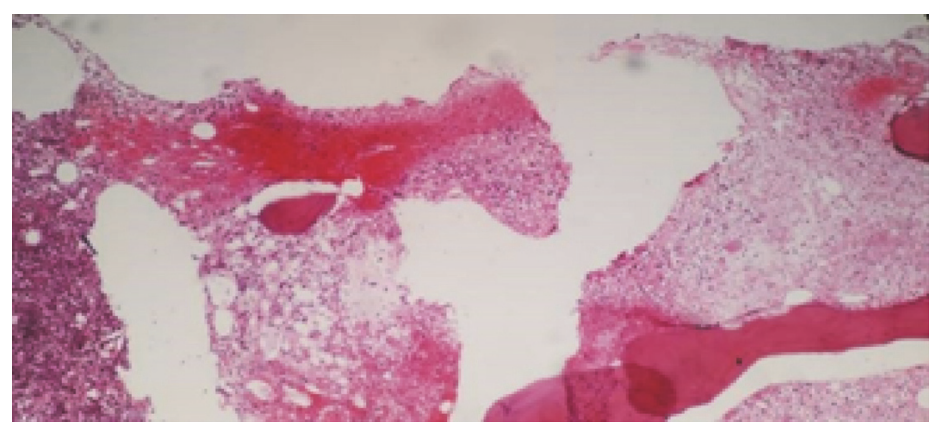

(b)

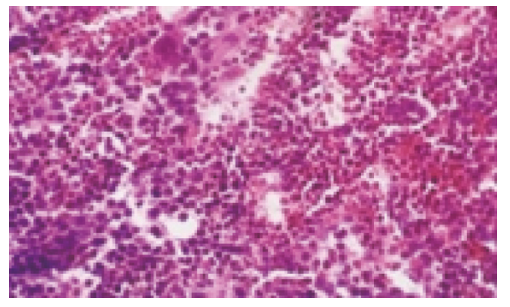

(d)

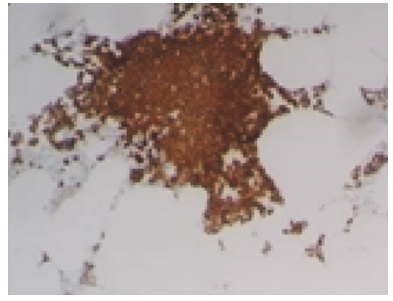

(e)

FIGURE 1: (a) Bone marrow aspirate showing an amorphous necrotic material (Giemsa stain $\times 100$ ). (b) Extensive BMN in the trephine biopsy $(H \& E \times 4)$. (c) Dilated sinus full of sickle cells in a background of ill-defined eosinophilic material (H\&E $\times 40)$. (d) Intact area with erythroid hyperplasia $(H \& E \times 40)$. (e) Spectrin stain showing the extent of erythroid hyperplasia $($ IHC, spectrin $\times 10)$.

\section{Case Presentation}

A 26-year-old Saudi female with known diabetes mellitus (DM) type-1 was admitted with jaundice, generalized body aches, and abdominal distention. She gave a history of chest infection that has been treated with antibiotics three days prior to her presentation. The family denied any previous hospitalizations or similar episodes in the past. Her mother and father are second-degree relatives with a history of sickle cell trait (SCT) in the father and DM type-1 in her identical twin sister. On examination the patient was conscious, alert, jaundiced, and not in distress and looked pale. Her vital signs were normal with clear chest examination and had a normal cardiovascular examination. Abdominal examination showed hepatosplenomegaly with ascites and she had intact central nervous system (CNS) examination. The initial blood count showed severe anemia with hemoglobin $(\mathrm{Hb})$ of $5 \mathrm{~g} / \mathrm{dL}$ (normal range $12.5-18 \mathrm{~g} / \mathrm{dL}$ ) and thrombocytopenia with platelet count of $5 \times 10^{9} / \mathrm{L}$ (normal range $150-450 \times 10^{9} / \mathrm{L}$ ). Reticulocyte count was $4.2 \%$ (normal range $0.5-2.5 \%$ ). PBF showed polychromasia with a marked leucoerythroblastic picture. Serum $\mathrm{LDH}$ was markedly elevated to $3000 \mathrm{U} / \mathrm{L}$ (normal range 135-255 U/L) and high total bilirubin 3.1 g/dL (normal range 0-1.2 g/dL), mainly indirect bilirubin. Renal function and other liver enzymes, however, were within the normal range. Computed tomography (CT) scan showed hepatosplenomegaly. Virology screen for corona, H1N1, and parvo virus was negative. During admission, the patient's condition deteriorated with declines in $\mathrm{Hb}$ and platelet count and an increase in serum LDH level. The patient was admitted to the intensive care unit (ICU) and bone marrow examination was done to exclude bone marrow infiltration. PBF after transfusion of 10 units of packed red blood cells (PRBC) showed a leucoerythroblastic picture with polychromasia and rare sickle cells. Bone marrow aspirate showed gelatinous basophilic material with distorted morphology. The bone marrow biopsy revealed extensive bone marrow necrosis involving more than half of the one centimeter length biopsy, markedly increased erythropoietic activity in the intact area, and dilated sinusoids which were full of sickled red blood cells (Figure 1). The diagnosis of extensive bone marrow necrosis secondary to sickle cell disease was reported based on these findings. $\mathrm{Hb}$ electrophoresis after transfusion showed $\mathrm{HbS}$ level of $34 \%$. Family studies were done and revealed that both parents had SCT and her identical twin was found to have SCD with a HbS level of $75 \%$. The patient received supportive therapy and eventually did very well. She subsequently received full vaccinations and began regular follow-up in the hematology clinic. Five months later her $\mathrm{Hb}$ electrophoresis showed a HbS level of $80 \%$.

\section{Discussion}

BMN is infrequently encountered in clinical practice. The incidence of BMN varies from 0.3 to $37 \%$ among different reports [11]. Such variability in the results could be attributed to the difference in the type of specimens examined (in vivo or postmortem), pathologist experience, and diagnostic criteria used (the incidence was reduced to 0.3 to $12 \%$ when only those biopsies with more than half bone marrow involvement by necrosis were included) [11]. BMN is defined as necrosis of hematopoietic tissue and stroma with preservation of cortical bone $[2,4]$. It has been identified with various clinical 
conditions including malignancy, infection, autoimmune disease, chemotherapy, DIC, anorexia nervosa, antiphospholipid syndrome, and sickle cell disease [3-6, 8-10].

Although the first case of BMN was reported in SCD patient, the association of BMN with SCD was reported in only $2 \%$ of the cases [4]. One possible cause of the paucity of this association is that bone marrow examination is not commonly done during sickle cell crisis [12]. Charache and Page stated that one of six patients with SCD has some degree of BMN during painful crisis; usually these patients have full recovery [4]. The largest review on BMN in SCD was done by Tsitsikas et al. who identified 58 cases of BMN with fat embolization syndrome (FES) and 16 cases of BMN without FES. In both groups there were a number of patients who were not known to have SCD prior to the presentation of BMN $19(33 \%)$ and $4(25 \%)$, respectively. It has been found that patients with genotype SS were at low risk for BMN/FES and, paradoxically, those with mild phenotypes were at higher risk of this catastrophic complication [10].

SCD is a relatively common genetic disorder in Saudi Arabia with the highest prevalence noticed in eastern province (approximately 21\% for SCT and 2.6\% for SCD) [13]. There are two major clinical phenotypes of SCD in Saudis. Patients from the western province have the severe phenotype which is consistent with the Benin haplotype. Acute chest syndrome with recurrence, stroke, dactylitis, lower base line total hemoglobin and hemoglobin F level, and early presentation with painful crisis are common clinical features of SCD in the western province. On the other hand patients with SCD from the eastern province have a more benign phenotype which is consistent with the Arab/Indian haplotype. They have a greater incidence of associated deletional alpha thalassemia, higher total hemoglobin and hemoglobin F levels, persistent splenomegaly, more avascular necrosis of the femoral head, and later disease presentation [14].

In our case, the first clinical disease presentation of SCD was extensive bone marrow necrosis with crisis at age of 26 years. She was not suspected to have the SCD prior to her presentation. She had never complained of bone pain and $\mathrm{Hb}$ electrophoresis had never been previously performed. Her identical twin sister as well was not known to have the disease nor did she complain of bone pain prior to the family study. Although the presentation of SCD in the eastern province of Saudi Arabia is relatively late as compared with the western province, presentation at this age (26 years old) in our patient and her identical twin is thought to be extremely uncommon. Also, the initial disease presentation with development of extensive BMN is rarely reported in the literature [15-18]. Having a mild SCD phenotype, the limited family history and lack of neonatal or later screening for hemoglobinopathies all contributed to the late diagnosis of SCD and postponed interventions that could possibly prevent life threatening complications of the disease such as BMN. This case presentation emphasizes the importance of SCD screening and need for follow-up of the patients as catastrophic complications could occur irrespective of the disease phenotype. This is especially important in an area with high SCD prevalence such as the eastern province of Saudi Arabia.
Another unique finding in our case is that she has coexistence of SCD and type-1 DM which is rarely reported as well in the literature $[19,20]$. Presence of the high prevalence of both diseases in this part of the world could provide a logical explanation for the coexistence of these disorders in these identical twin sisters.

\section{Conclusions}

$\mathrm{BMN}$ is infrequently encountered in clinical practice and rarely reported in association with SCD. As the initial presentation of SCD, BMN was previously reported only in a few cases in the literature. Patients with a mild SCD phenotype are at high risk of BMN. Thus, a high index of suspicion must be borne in mind to prevent the development of catastrophic complications particularly, in an area with a high prevalence of SCD.

\section{Consent}

Written informed consent was obtained from the patient for publication of this case report and any accompanying images.

\section{Conflicts of Interest}

The authors declare that they have no conflicts of interest.

\section{Authors' Contributions}

Sameera A. Alsafwani, Abdulwahed Al-Saeed, and Rehab Bukhamsin were involved actively in the management of the patient. Sameera A. Alsafwani drafted the manuscript. All the others provided valuable inputs and guidance during the preparation of the manuscript. All authors read and approved the final manuscript.

\section{Acknowledgments}

The authors of this article acknowledged the hematopathology consultants in DRL, Mariam Alghazal, Azza Abualam, and Mohamed Khan, for their opinions in writing the bone marrow report.

\section{References}

[1] L. J. Wade and L. D. Stevenson, "Necrosis of bone marrow with fat embolism in sickle cell anemia," American Journal of Pathology, vol. 17, pp. 47-54, 1941.

[2] D. Maisel, J. Y. Lim, W. J. Pollock, R. Yatani, and P. I. Liu, "Bone marrow necrosis: an entity often overlooked," Ann Clin Lab Sci, vol. 18, pp. 109-115, 1988.

[3] S. Paydas, M. Ergin, F. Baslamisli et al., "Bone marrow necrosis: clinicopathologic analysis of 20 cases and review of the literature," American Journal of Hematology, vol. 70, no. 4, pp. 300305, 2002.

[4] A. M. Janssens, F. C. Offner, and W. Z. Van Hove, "Bone marrow necrosis," Cancer, vol. 88, no. 8, pp. 1769-1780, 2000.

[5] L. Ding, A. Rawal, S. Luikart, and P. Wadhwa, "Necrosis of uninvolved bone marrow following filgrastim administration in 
a patient with Burkitt lymphoma undergoing chemotherapy," British Journal of Haematology, vol. 159, no. 1, p. 2, 2012.

[6] K. Foucar, K. Reichard, and D. Czuchlewski, Bone Marrow Pathology, ASCP, Chicago, IL, USA, 3rd edition, 2010.

[7] A. Almeida and I. Roberts, "Bone involvement in sickle cell disease," British Journal of Haematology, vol. 129, no. 4, pp. 482490, 2005.

[8] P. Ricci, G. Bandini, M. Baccarani, F. Bazzocchi, G. Martinelli, and S. Tura, "Bone marrow necrosis by diffuse metastatic intravascular obstruction,” Haematologica, vol. 67, no. 5, pp. 754-759, 1982.

[9] C. Knupp, P. H. Pekala, and P. Cornelius, "Extensive bone marrow necrosis in patients with cancer and tumor necrosis factor activity in plasma," American Journal of Hematology, vol. 29, no. 4, pp. 215-221, 1988.

[10] D. A. Tsitsikas, G. Gallinella, S. Patel, H. Seligman, P. Greaves, and R. J. Amos, "Bone marrow necrosis and fat embolism syndrome in sickle cell disease: Increased susceptibility of patients with non-SS genotypes and a possible association with human parvovirus B19 infection," Blood Reviews, vol. 28, no. 1, pp. 23-30, 2014.

[11] T. C. S. Cabral, C. M. Fernandes, L. A. C. Lage, M. C. Zerbini, and J. Pereira, "Bone marrow necrosis: literature review," Jornal Brasileiro de Patologia e Medicina Laboratorial, vol. 52, no. 3, pp. 182-188, 2016.

[12] P. D. Ziakas and M. Voulgarelis, "Bone marrow necrosis in sickle cell anaemia," Blood Transfusion, vol. 8, no. 3, p. 211, 2010.

[13] Z. Naserullah, A. Alshammari, M. Al Abbas et al., "Regional experience with newborn screening for sickle cell disease, other hemoglobinopathies and G6PD deficiency," Annals of Saudi Medicine, vol. 23, no. 6, pp. 354-357, 2003.

[14] W. Jastaniah, "Epidemiology of sickle cell disease in Saudi Arabia," Annals of Saudi Medicine, vol. 31, no. 3, pp. 289-293, 2011.

[15] M. Shafiq and N. Ali, "Bone marrow necrosis-initial presentation in sickle cell anaemia," American Journal of Case Reports, vol. 14, pp. 416-418, 2013.

[16] G. El Hachem and N. Chamseddine, "Bone marrow necrosis: an unusual initial presentation of sickle cell anemia," Clinical Lymphoma, Myeloma and Leukemia, vol. 16, pp. S195-S196, 2016.

[17] J. Adamski, C. A. Hanna, V. B. Reddy, S. H. Litovsky, C. A. Evans, and M. B. Marques, "Multiorgan failure and bone marrow necrosis in three adults with sickle cell- $\beta^{+}$-thalassemia," American Journal of Hematology, vol. 87, no. 6, pp. 621-624, 2012.

[18] F. Simon, E. Carloz, B. Chaudier, P. Kraemer, P. Colbacchini, and P. Hovette, "Extensive bone marrow necrosis as presenting manifestation of sickle cell disease in Africa," Medecine tropicale : revue du Corps de sante colonial, vol. 64, no. 2, pp. 179-182, 2004.

[19] O. O. Jarrett and E. I. Olorundare, "Type 1 diabetes mellitus in a known sickle cell anaemia patient: a rare combination in Nigeria," African Journal of Medicine and Medical Sciences, vol. 43, no. 2, pp. 177-181, 2014.

[20] Z. Shoar, G. Rezvani, and F. De Luca, “Type 1 diabetes mellitus in a patient with homozygous sickle cell anemia," Journal of Pediatric Endocrinology and Metabolism, vol. 26, no. 11-12, pp. 1205-1207, 2013. 


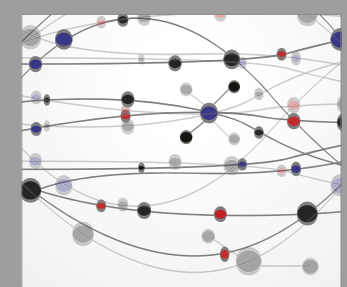

The Scientific World Journal
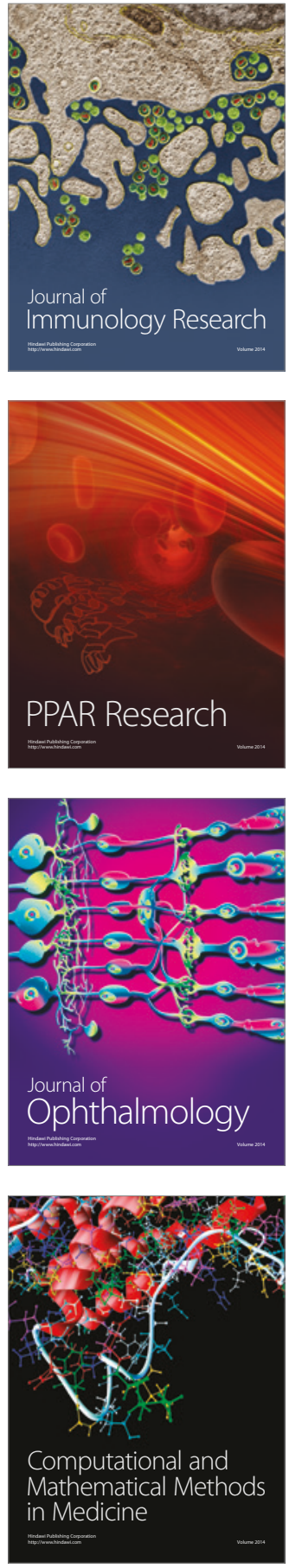

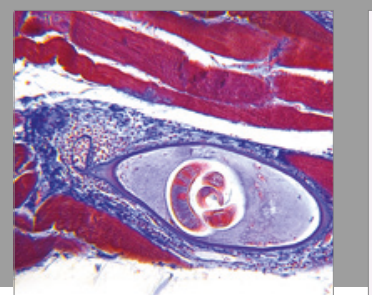

Gastroenterology Research and Practice
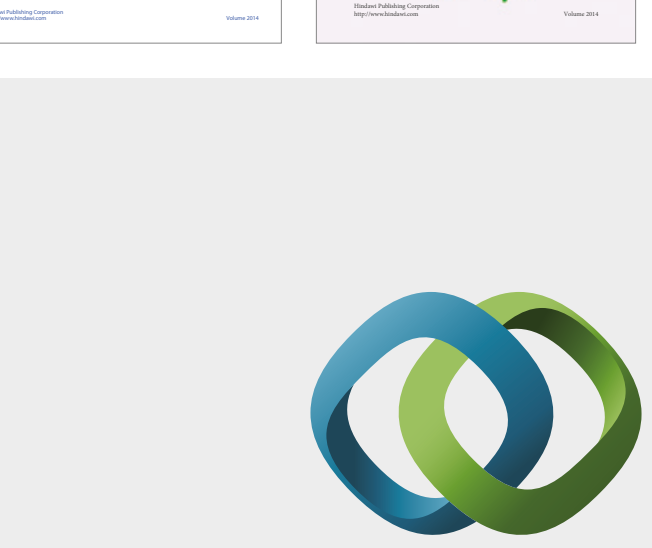

\section{Hindawi}

Submit your manuscripts at

https://www.hindawi.com
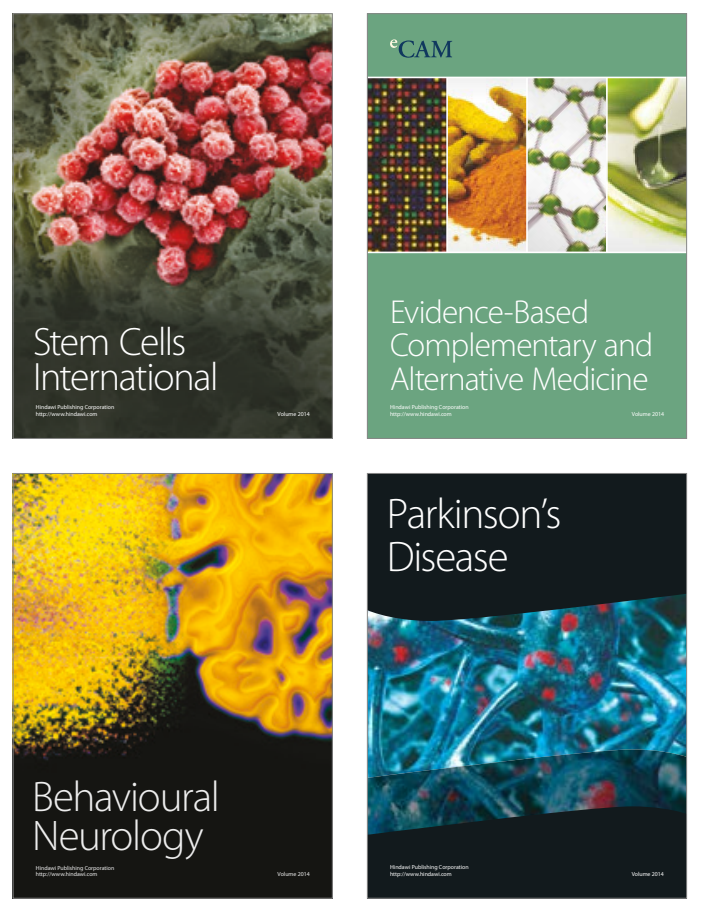
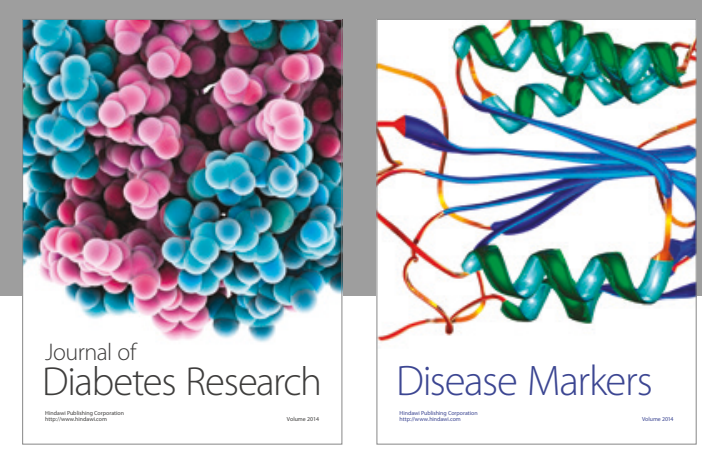

Disease Markers
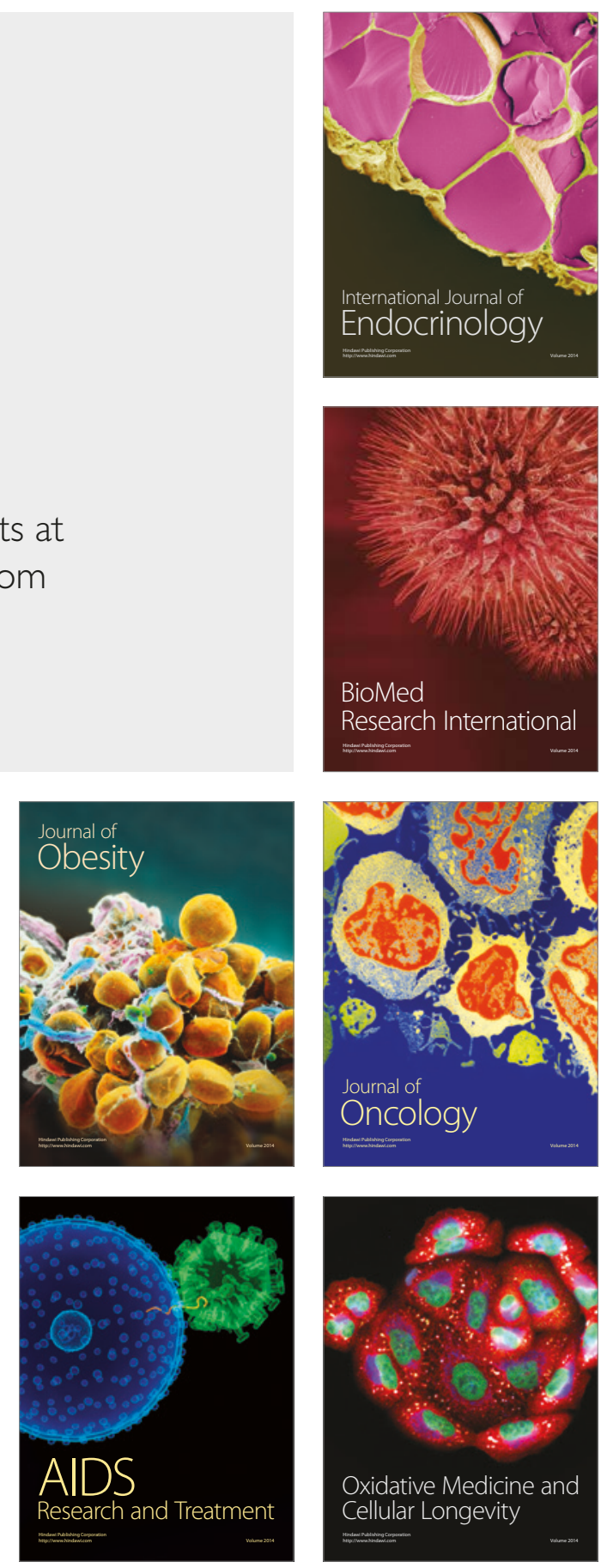